\title{
The correlation between primary dysmenorrhea and oxidative stress markers in adolescents
}

\author{
Bergen Laleli Koç ${ }^{1 \oplus,}$ Burcu Timur²® \\ ${ }^{1}$ Department of Obstetrics and Gynecology, Ankara City Hospital, Ankara, Turkey \\ ${ }^{2}$ Department of Obstetrics and Gynecology, Ordu University Training and Research Hospital, Ordu, Turkey
}

\begin{abstract}
Objectives: Primary dysmenorrhea is the most common gynecological complaint in adolescent girls. Among many factors, oxidative stress (OS) is thought to be a potential factor in the etiology of primary dysmenorrhea. In this study, it is thought that the use of IMA, thiol, and disulfide levels as a diagnostic marker in primary dysmenorrhea and antioxidant interventions may play a role in the treatment of primary dysmenorrhea and may benefit the pathophysiological and treatment process of the disease.

Methods: Thirty adolescent girls (study group) with grade 2.3 primary dysmenorrhea who applied to outpatient clinic and 30 healthy young girls (control group) of similar age group were included in the study. Primary dysmenorrhea grade was analyzed with the help of the visual analog scale (VAS) and verbal multidimensional scoring system (VMS). Patient's basal hormone levels in the early follicular phase, serum albumin, IMA, total thiol, native thiol, disulfide, C-reactive protein (CRP), and cancer antigen-125 (CA-125) were recorded as main parameters.
\end{abstract}

Results: Oxidative stress markers were compared between the primary dysmenorrhea and control groups. Although albumin, IMA, and disulfide levels were higher on average in the group with dysmenorrhea, the difference was not statistically significant. Disulfide level was found to be significantly higher in the group with CA125 $\geq 35$.

Conclusions: In our study, we examined serum albumin, IMA, total thiol, native thiol and disulfide levels in two groups resuts were not statistically significant. In this study, we concluded that as the CA125 level increased, the disulfide level increased in parallel.

Keywords: Adolescents, dysmenorrhea, oxidative stress

$\mathbf{P}$ rimary dysmenorrhea refers to recurrent and cramp-like pain in the lower abdomen that occurs during menstruation in the absence of a pelvic pathology. It is the most common gynecological complaint in adolescent girls [1]. Primary dysmenorrhea is more common in young women and women who have not given birth yet [2]. The management of the disease begins by ruling out a pelvic pathology. Dysmenorrhea accompanied by a pelvic pathology is called secondary dysmenorrhea. The most common cause is endometriosis. Although secondary dysmenorrhea is more common in women in the $4^{\text {th }}$ or $5^{\text {th }}$ decade of life, it may rarely occur in adolescence.

Primary dysmenorrhea is caused by the increased production of endometrial prostaglandins during menstruation. Due to increased prostaglandins, the basal 
tone and active pressure of the uterus and dysrhythmic contraction pattern increase. The decreased uterine blood flow due to uterine hypercontractility and increased sensitivity in peripheral nerves contribute to pain. It starts a few years after menarche, after the ovulatory cycles settle down, and although it is more common in young women, it can continue until the $40 \mathrm{~s}$. The prevalence in adolescents ranges from $60 \%$ to 93\% [3]. The pain is colic and begins a few hours before or with menstruation. Symptoms may be accompanied by nausea, vomiting, diarrhea, back pain, and syncope attacks. Early menarche, long menstrual periods, smoking, and high BMI may increase the severity and duration of pain.

Although the cause of primary dysmenorrhea is not fully understood, endometrial prostaglandins are thought to be associated with primary dysmenorrhea. It has been shown that PGF2alpha and PGF2alpha/PGE2 ratios increase in patients with primary dysmenorrhea, especially in the endometrium [4]. Prostaglandins are thought to cause pain by stimulating myometrial contractions, reducing uterine blood flow and oxygenation. During menstruation, when the uterus rises from the basal contraction level of $10 \mathrm{mmHg}$ to $150-180 \mathrm{mmHg}$, uterine ischemia develops, and the released anaerobic metabolites stimulate type $\mathrm{C}$ pain fibers and cause dysmenorrhea [5]. Treatment options for dysmenorrhea include NSAIDs, COCs, and non-pharmacological alternative treatment options (such as TENS, acupuncture, exercise, diet) [6].

Prostaglandins are released from the endometrium during menstruation, increasing intrauterine pressure, and uterine ischemia develops. Ischemia leads to proinflammatory reaction cascades that lead to reactive oxygen radical production. Oxidative stress (OS) is defined as an imbalance between free radicals that occur as a result of normal metabolism or pathologically in living organisms and the antioxidant defense system, which has a protective role against free radicals $[7,8]$. OS is thought to cause diseases by creating toxic effects on carbohydrate, protein, lipid, and DNA metabolism. Among many factors, OS is thought to be a potential factor in the etiology of primary dysmenorrhea. In the presence of OS, normal human serum albumin (HSA) undergoes modification and turns into ischemia-modified albumin (IMA) form with reduced affinity for metals such as cobalt, nickel, and copper
[9]. IMA is a metabolic variant resulting from ischemic conditions in serum albumin. Increased free levels of IMA are studied as a simple and new marker for OS, hypoxia, inflammation, and endothelial dysfunction in some endocrine disorders [10]. Dynamic thiol/disulfide homeostasis has also recently been identified as an oxidative stress marker and has been shown to contribute to antioxidant defense, detoxification, and apoptosis [11]. It is an organic component containing the thiol-SH group and is an important component of the antioxidant system. The primary target of reactive oxygen radicals (ROS) is - $\mathrm{SH}$ groups of sulfur-containing amino acids, and the first action of ROS is to convert -SH groups into reversible disulfide bonds by oxidation. Disulfide bonds can be reduced back to thiol groups, and in this way, thiol/disulfide homeostasis is achieved [12].

This study aimed to evaluate uterine ischemia occurring during dysmenorrhea with oxidative stress markers such as IMA, thiol/disulfide. In this study, it is thought that the use of IMA, thiol, and disulfide levels as a diagnostic marker in primary dysmenorrhea and antioxidant interventions may play a role in the treatment of primary dysmenorrhea and may benefit the pathophysiological and treatment process of the disease.

\section{METHODS}

\section{Patient Selection and Study Protocol}

Thirty adolescent girls (study group) with grade 2.3 primary dysmenorrhea who applied to the University of Health Sciences Zekai Tahir Burak Health Application and Research Center youth center polyclinic between April 2019 and August 2019, and 30 healthy young girls (control group) of similar age group, who did not have any additional disease and did not have primary dysmenorrhea, were included in the study. Those with endocrinological (adrenal hyperandrogenism, Cushing's syndrome, DM) and chronic disease and those who used drugs were excluded from the study.

All patients underwent general physical and pelvic examination, detailed anamnesis was obtained, and sociodemographic characteristics were recorded. Primary dysmenorrhea grade was analyzed with the help of the visual analog scale (VAS) and verbal multidi- 
mensional scoring system (VMS). Patients' age, body mass index (BMI), waist circumference, hip circumference measurements, waist/hip ratios, age at menarche, menstrual patterns, basal hormone levels in the early follicular phase, serum albumin, IMA, total thiol, native thiol, disulfide, $\mathrm{C}$-reactive protein (CRP), and cancer antigen-125 (CA-125) were recorded as main parameters. The ovarian and uterus imaging of the patients was performed by abdominal ultrasonography. Blood samples were taken from the antecubital vein (approximately $10 \mathrm{ml}$ ) on the morning of the $2^{\text {nd }}$ or $3^{\text {rd }}$ day of menstruation for basal hormone levels and after 8 hours of night fasting for the evaluation of biochemical parameters, and the samples were taken to the laboratory within 10 minutes.

Thiol and disulfide and IMA levels were analyzed by a newly developed method by Erel and Kosem [11]. In summary, the reducible disulfide linkages were reduced to free functional thiol groups. Half of the difference between total and native thiol provides the dynamic disulfide amount. After determining the amount of native thiol and disulfide, the ratio of native thiol/disulfide was measured. Measurement analyses were performed blindly to the clinical information and course of the patients, and results were not available to the treating clinician, study group, or researchers during the study period.

Ethical approval was obtained (Date: 16.01.2019, Decision No:11/2019) from Dr. Zekai Tahir Burak Education and Research Hospital and the study was conducted in accordance with the Helsinki Declaration.

\section{Statistical Analysis}

The Statistical Package for the Social Sciences
(SPSS) 23 program was used in the data analysis. The variables were investigated using visual (histograms, probability plots) and analytical methods (Kolmogorov-Simirnov/Shapiro-Wilk's test) to determine whether or not they were normally distributed. Descriptive analyses were presented using means and standart deviations for normally distributed variables. Parametric methods were used in the analysis of normally distributed variables. The independent samples T-test was used in the comparison of two independent groups. A p-value of less than 0.05 was considered to show a statistically significant result.

\section{RESULTS}

The study was conducted between April 2019 and August 2019 on a total of 60 patients aged between 15-22 years. Albumin, IMA, thiol, and disulfide levels were compared between the primary dysmenorrhea and control groups. Although albumin, IMA, and disulfide levels were higher on average in the group with dysmenorrhea, the difference was not statistically significant $(p>0.05)$. Although native thiol and total thiol levels were lower on average in the group with dysmenorrhea, the difference was not statistically significant $(p>0.05)$ (Table 1).

Anthropometric characteristics of the cases are shown. Of the 60 cases, 30 had primary dysmenorrhea, and 30 were healthy individuals who applied to the youth center polyclinic. The mean age of the dysmenorrhea patients was $19.63 \pm 2.93$ years, and the mean age of the control group was $18.33 \pm 2.56$ years, and there was no statistical difference. Body mass index,

Table 1. Comparison of oxidative stress markers between the groups

\begin{tabular}{lccc}
\hline & $\begin{array}{c}\text { Dysmenorrhea group } \\
(\mathbf{n}=\mathbf{3 0}) \\
\text { Mean } \pm \text { SD }\end{array}$ & $\begin{array}{c}\text { Control group } \\
(\mathbf{n}=\mathbf{3 0}) \\
\text { Mean } \pm \text { SD }\end{array}$ & $p$ value \\
\hline Albumin & $4.29 \pm 0.09$ & $4.26 \pm 0.1$ & 0.211 \\
Albumin adjusted IMA & $0.98 \pm 0.2$ & $0.96 \pm 0.21$ & 0.777 \\
IMA & $0.98 \pm 0.2$ & $0.97 \pm 0.2$ & 0.858 \\
Native Thiol & $409.52 \pm 26.14$ & $414.99 \pm 29.49$ & 0.450 \\
Total Thiol & $449.67 \pm 29.13$ & $459.1 \pm 32.3$ & 0.240 \\
Disulphide & $22.32 \pm 3.45$ & $21.0 \pm 2.64$ & 0.108 \\
\hline
\end{tabular}

IMA $=$ ischemia-modified albumin 
Tablo 2. Anthropometric characteristics of dysmenorrhea and control cases

\begin{tabular}{lccc}
\hline & $\begin{array}{c}\text { Dysmenorrhea group } \\
(\mathbf{n}=\mathbf{3 0}) \\
\text { Mean } \pm \text { SD }\end{array}$ & $\begin{array}{c}\text { Control group } \\
(\mathbf{n}=\mathbf{3 0}) \\
\text { Mean } \pm \text { SD }\end{array}$ & $p$ value \\
\hline Age $($ years $)$ & $19.63 \pm 2.93$ & $18.33 \pm 2.56$ & 0.073 \\
BMI $\left(\mathrm{kg} / \mathrm{m}^{2}\right)$ & $21.28 \pm 2.75$ & $21.21 \pm 3.40$ & 0.937 \\
Waistline $(\mathrm{cm})$ & $70.20 \pm 7.99$ & $70.10 \pm 9.85$ & 0.966 \\
Hip circumference $(\mathrm{cm})$ & $96.30 \pm 6.96$ & $95.20 \pm 7.55$ & 0.560 \\
Hip/waist ratio & $0.72 \pm 0.04$ & $0.73 \pm 0.05$ & 0.832 \\
\hline BMI & & &
\end{tabular}

$\mathrm{BMI}=$ body mass index

waist circumference, hip circumference, and waist/hip ratios were similar between the two groups $(p>0.05)$ (Table 2).

When OS markers were compared in the smoker and non-smoker groups, statistically similar values were measured $(p>0.05)$. Smoking did not cause any change in the level of OS markers (Table 3 ).
When OS markers were compared according to the CA-125 level, disulfide level was found to be significantly higher in the group with CA125 $\geq 35$ ( $p<$ $0.05)$. Other OS markers were found to be similar between the groups (Table 4).

OS markers were compared between patients with a primary dysmenorrhea severity of VMS grade 2 and

Tablo 3. Comparison of OS markers in smoker and non-smoker groups

\begin{tabular}{lccc}
\hline & $\begin{array}{c}\text { Smoker } \\
(\mathbf{n}=\mathbf{6}) \\
\text { Mean } \pm \text { SD }\end{array}$ & $\begin{array}{c}\text { Non-smoker } \\
(\mathbf{n}=\mathbf{5 4}) \\
\text { Mean } \pm \text { SD }\end{array}$ & $p$ value \\
\hline Albumin & $4.24 \pm 0.14$ & $4.28 \pm 0.08$ & 0.357 \\
Albumin adjusted IMA & $0.87 \pm 0.05$ & $0.98 \pm 0.21$ & 0.212 \\
\hline IMA & $0.88 \pm 0.06$ & $0.98 \pm 0.20$ & 0.239 \\
Native thiol & $397.31 \pm 31.40$ & $413.91 \pm 27.15$ & 0.167 \\
Total thiol & $437.70 \pm 32.06$ & $456.24 \pm 30.47$ & 0.165 \\
\hline Disulphide & $21.64 \pm 3.13$ & $21.67 \pm 3.14$ & 0.688 \\
\hline
\end{tabular}

IMA $=$ ischemia-modified albümin, $\mathrm{OS}=$ oxidative stress

Table 4. Comparison of OS markers according to the CA-125 level

\begin{tabular}{lccc}
\hline & $\begin{array}{c}\text { CA125 }<\text { 35 } \\
\text { Mean } \pm \text { SD }\end{array}$ & $\begin{array}{c}\text { CA 125 } \geq \mathbf{3 5} \\
\text { Mean } \pm \text { SD }\end{array}$ & p value \\
\hline Albumin & $4.27 \pm 0.09$ & $4.31 \pm 0.09$ & 0.241 \\
Albumin adjusted IMA & $0.96 \pm 0.20$ & $1.02 \pm 0.17$ & 0.441 \\
IMA & $0.96 \pm 0.20$ & $1.02 \pm 0.17$ & 0.513 \\
Native thiol & $413.55 \pm 28.10$ & $402.41 \pm 24.65$ & 0.323 \\
Total thiol & $454.64 \pm 31.51$ & $452.40 \pm 27.53$ & 0.858 \\
Disulphide & $21.35 \pm 3.00$ & $24.12 \pm 3.06$ & $\mathbf{0 . 0 2 6}$ \\
\hline
\end{tabular}

IMA $=$ ischemia-modified albümin, OS = oxidative stress, CA $125=$ cancer antigen 125 
Table 5. Evaluation of OS markers according to the VMS score

\begin{tabular}{lccc}
\hline & $\begin{array}{c}\text { VMS Grade 2 } \\
\text { Mean } \pm \text { SD }\end{array}$ & $\begin{array}{c}\text { VMS Grade 3 } \\
\text { Mean } \pm \text { SD }\end{array}$ & p value \\
\hline Albumin & $4.29 \pm 0.09$ & $4.28 \pm 0.90$ & 0.648 \\
Albumin adjusted IMA & $0.99 \pm 0.21$ & $0.93 \pm 0.16$ & 0.515 \\
\hline IMA & $0.99 \pm 0.21$ & $0.94 \pm 0.16$ & 0.554 \\
Native thiol & $411.26 \pm 26.74$ & $405.45 \pm 25.74$ & 0.586 \\
Total thiol & $449.32 \pm 28.41$ & $450.47 \pm 32.51$ & 0.923 \\
Disulphide & $22.25 \pm 2.14$ & $22.47 \pm 5.62$ & 0.873 \\
\hline
\end{tabular}

IMA $=$ ischemia-modified albümin, $\mathrm{OS}=$ oxidative stress, $\mathrm{VMS}=$ verbal multidimensional scoring system

grade 3 . As the severity of dysmenorrhea increased, the level of OS markers did not change statistically (Table 5).

\section{DISCUSSION}

Although OS is associated with primary dysmenorrhea, studies on the subject are limited. The end products of OS and associated metabolites have become targets in therapeutic approaches for diseases associated with intense inflammation and endothelial dysfunction [13].

The menstruation of the participants aged between 15 and 22 and included in the study was regular, the mean age at menarche was 12 in both groups, the mean length of menstruation period was 5.8 days in the dysmenorrhea group and 6.2 days in the control group. Although serum albumin, IMA, and disulfide levels were higher on average in the group with dysmenorrhea, the difference was not statistically significant. Although the native thiol and total thiol levels were lower on average in the group with dysmenorrhea, the difference was not statistically significant. There is no other study in the literature evaluating these markers, which are indicators of oxidative stress in patients with primary dysmenorrhea. Therefore, our study is the first one in this regard. However, there are other studies in the literature stating that OS markers increase in primary dysmenorrhea. For example, in a study examining lipid and protein peroxidation markers among university students with dysmenorrhea in Nigeria, malondialdehyde (MDA) values were significantly higher in the group with dysmenorrhea [14]. This study also supports the studies conducted by
Turhan et al. [15] and Dikensoy et al. [16]. This study carried out on Nigerian women also determined higher plasma nitrotyrosine (3-NT) levels and lower plasma alpha tocopherol (vitamin E) levels in the dysmenorrhea group. Protein carbonyls (PrCarb) levels did not differ between the groups [14]. While Turhan et al. [15], in their study investigating the oxidative stress balance in patients with primary dysmenorrhea using multiple serum markers, found the malondialdehyde (MDA) level to be high in the dysmenorrhea group as stated above, they found nitrotyrosine (3-NT), deoxyguanosine (8-OHdG) and superoxide dismutase (SOD) levels to be similar between the groups. They also detectedno correlation between the level of oxidative stress markers and the severity of dysmenorrhea [15]. In the study performed by Dikensoy et al. [16], malondialdehyde (MDA), nitric oxide (NO), and adrenomedullin (AM) levels were found to be significantly higher in the dysmenorrhea group. They concluded that lipid peroxidation and oxidative stress played an important role in the etiopathogenesis of primary dysmenorrhea [16].

When the anthropometric characteristics of dysmenorrhea and control cases were compared, the mean BMI was $21 \%$, the mean waist circumference was 70 $\mathrm{cm}$, the waist/hip ratios were similar in both groups, and no difference was found between the groups. It was reported that menstruation in adolescents did not show a significant correlation with body mass index [17]. In a study investigating the factors affecting the severity of dysmenorrhea, it was concluded that height, weight, and menstrual cycle length were not associated with the severity of dysmenorrhea, but there was a strong correlation with age at menarche and smoking. It was determined that the prevalence of 
dysmenorrhea was higher and the symptoms were more severe in smoking women $[18,19]$. In our study, we could not determine a correlation between the severity of dysmenorrhea and smoking, as we did not detect a difference in the level of OS markers in the smoker and non-smoker group, in contrast to the findings in the above-mentioned study. At the same time, when we evaluated the severity of dysmenorrhea by grading with the VMS score, we found that the severity of dysmenorrhea and the levels of OS markers did not change. Furthermore, although albumin, IMA, and disulfide levels were higher in the group with CA 125 $\geq 35$ than the group with low CA-125, the difference between only disulfide levels was statistically significant.

\section{Limitations}

The small number of patients included in the study (30 patients with dysmenorrhea, 30 controls) and the lack of quantitative diagnostic criteria to define dysmenorrhea are the weaknesses of our study and are likely to have affected our results. The place of the level of OS markers in the diagnosis of dysmenorrhea should be supported by studies involving larger patient groups and adult women of reproductive age.

\section{CONCLUSION}

Since primary dysmenorrhea is a gynecological problem experienced by many young women and significantly affects their quality of life, it is reasonable to investigate $\mathrm{OS}$ in the etiopathogenesis of primary dysmenorrhea. The idea of controlling painful menstruation by reducing OS is important in this sense. In our study, in which we examined serum albumin, IMA, total thiol, native thiol, and disulfide levels in 30 women with dysmenorrhea and 30 healthy adolescents and young adults, changes were determined in the level of OS markers in the dysmenorrhea group compared to the control group, which was consistent with the literature, but not statistically significant. In this study, we concluded that as the CA125 level increased, the disulfide level increased in parallel. There is no other study on dysmenorrhea patients in the literature that investigates the OS markers we studied.

\section{Authors' Contribution}

Study Conception: BLK; Study Design: BLK; Supervision: BLK; Funding: BLK; Data Collection and/or Processing: BLK; Statistical Analysis and/or Data Interpretation: BT; Literature Review: BT; Manuscript Preparation: BLK and Critical Review: BT.

\section{Conflict of interest}

The authors disclosed no conflict of interest during the preparation or publication of this manuscript.

\section{Financing}

The authors disclosed that they did not receive any grant during conduction or writing of this study.

\section{REFERENCES}

1. Wilson CA, Keye WR, Jr. A survey of adolescent dysmenorrhea and premenstrual symptom frequency. A model program for prevention, detection, and treatment. J Adolesc Health Care 1989; 10:317-22.

2. Pawlowski B. Prevalence of menstrual pain in relation to the reproductive life history of women from the Mayan rural community. Ann Human Biol 2004;31:1-8.

3. Hillen TI, Grbavac SL, Johnston PJ, Straton JA, Keogh JM. Primary dysmenorrhea in young Western Australian women: prevalence, impact, and knowledge of treatment. J Adolesc Health 1999;25:40-5.

4. Willman EA, Collins WP, Clayton SG. Studies in the involvement of prostaglandins in uterine symptomatology and pathology. Br J Obstet Gynaecol 1976;83:337-41.

5. Dawood MY. Primary dysmenorrhea: advances in pathogenesis and management. Obstet Gynecol 2006;108:428-41.

6. Campbell MA, McGrath PJ. Use of medication by adolescents for the management of menstrual discomfort. Arch Pediatr Adolesc Med 1997;151:905-13.

7. McCord JM. Human disease, free radicals, and the oxidant/antioxidant balance. Clin Biochem 1993;26:351-7.

8. Ustundag Y, Huysal K, Kahvecioglu S, Demirci H, Yavuz S, Sambel M, et al. Establishing reference values and evaluation of an in-house ferric reducing antioxidant power (FRAP) colorimetric assay in microplates. Eur Res J 2016;2:126-31.

9. Bar-Or D, Rael LT, Bar-Or R, Slone DS, Mains CW, Rao NK, et al. The cobalt-albumin binding assay: insights into its mode of action. Clin Chim Acta 2008;387:120-7.

10. Ustun Y, Engin-Ustun Y, Ozturk O, Alanbay I, Yaman H. Ischemia-modified albumin as an oxidative stress marker in preeclampsia. J Matern Fetal Neonatal Med 2011;24:418-21.

11. Erel O, Neselioglu S. A novel and automated assay for thiol/disulphide homeostasis. Clin Biochem 2014;47:326-32.

12. Dean RT, Fu S, Stocker R, Davies MJ. Biochemistry and pathology of radical-mediated protein oxidation. Biochem $\mathrm{J}$ 1997;324(Pt 1):1-18.

13. Kaplan O, Naziroglu M, Guney M, Aykur M. Non-steroidal 
anti-inflammatory drug modulates oxidative stress and calcium ion levels in the neutrophils of patients with primary dysmenorrhea. J Reprod Immunol 2013;100:87-92.

14. Orimadegun BE, Awolude OA, Agbedana EO. Markers of lipid and protein peroxidation among Nigerian university students with dysmenorrhea. Niger J Clin Pract 2019;22:174-80.

15. Turhan N, Celik H, Duvan CI, Onaran Y, Aydin M, Armutcu F. Investigation of oxidative balance in patients with dysmenorrhea by multiple serum markers. J Turk German Gynecol Assoc 2012;13:233-6.

16. Dikensoy E, Balat O, Pence S, Balat A, Cekmen M, Yurekli
M. Malondialdehyde, nitric oxide and adrenomedullin levels in patients with primary dysmenorrhea. J Obstet Gynaecol Res 2008;34:1049-53.

17. Nafstad P, Stray-Pedersen B, Solvberg M, Tangen T. [Menarche and menstruation problems among teenagers in Oslo 1993]. Tidsskr Nor Laegeforen 1995;115:604-6. [Article in Norwegian] 18. Sundell G, Milsom I, Andersch B. Factors influencing the prevalence and severity of dysmenorrhoea in young women. $\mathrm{Br}$ J Obstet Gynaecol 1990;97:588-94.

19. Pullon S, Reinken J, Sparrow M. Prevalence of dysmenorrhoea in Wellington women. N Z Med J 1988;101:52-4. 